\title{
Tenofovir-Associated Bone Adverse Outcomes among a US National Historical Cohort of HIV- Infected Veterans: Risk Modification by Concomitant Antiretrovirals
}

Joanne LaFleur · Adam P. Bress · Joel Myers · Lisa Rosenblatt •

Jacob Crook · Kristin Knippenberg · Roger Bedimo • Pablo Tebas •

Heather Nyman · Stephen Esker

Received: August 25, 2017 / Published online: February 28, 2018

(C) The Author(s) 2018. This article is an open access publication

\section{ABSTRACT}

Introduction: Tenofovir disoproxil fumarate (TDF) has been associated with greater incidences of bone complications, which might be modified by some concomitantly administered antiretrovirals, possibly by their effect on tenofovir concentrations. We compared bone adverse outcomes among treatment-naïve HIVinfected US veterans initiating efavirenz (EFV)containing TDF/emtricitabine (FTC) regimens

Enhanced Content To view enhanced content for this article go to https://doi.org/10.6084/m9.figshare. 5887165 .

Electronic supplementary material The online version of this article (https://doi.org/10.1007/s40121018-0194-1) contains supplementary material, which is available to authorized users.

J. LaFleur $(\bowtie) \cdot$ K. Knippenberg $\cdot$ H. Nyman Department of Pharmacotherapy, University of Utah, Salt Lake City, UT, USA

e-mail: Joanne.Lafleur@pharm.utah.edu

J. LaFleur · A. P. Bress · J. Crook · K. Knippenberg Salt Lake City VA Health Care System, Salt Lake City, UT, USA

\section{A. P. Bress}

Department of Population Health Sciences, University of Utah School of Medicine, Salt Lake City, UT, USA

J. Myers · L. Rosenblatt · S. Esker Bristol-Myers Squibb, Lawrenceville, NJ, USA versus those initiating non-EFV-containing TDF/FTC regimens.

Methods: Using national Veterans Health Administration clinical and administrative data sets, we identified a cohort of treatment-naïve HIV-infected veterans without bone disease who initiated therapy with TDF/FTC plus EFV, rilpivirine, elvitegravir/cobicistat, or ritonavirboosted protease inhibitors in 2003-2015. The primary composite adverse bone outcome was the unadjusted incidence rate (IR) of osteoporosis, osteopenia, or fragility fracture (any hip, wrist, or spine fracture). To account for selection bias and confounding, we used inverse probability of treatment-weighted Cox proportional hazards regression models to calculate adjusted hazard ratios (HRs) for each outcome associated with EFV + TDF/FTC versus each non-EFV-containing TDF/FTC regimen.

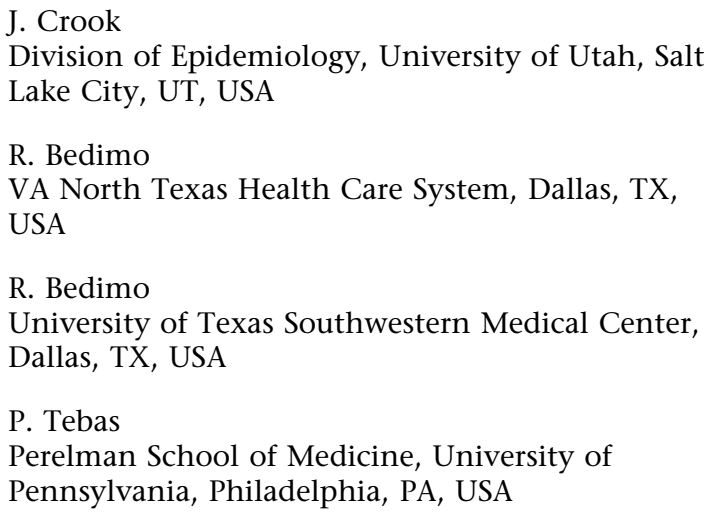


Results: Of 33,048 HIV-positive veterans, 7161 initiated a TDF/FTC-containing regimen (mean age, 50 years; baseline CD4 $<200$ cells $/ \mathrm{mm}^{3}$, $33.3 \%$; HIV-1 RNA > 100,000 copies/ml, 22.3\%; mean follow-up, 13.0 months). Of these, 4137 initiated EFV- and 3024 non-EFV-containing regimens. Veterans initiating EFV- versus nonEFV-containing TDF/FTC regimens had a lower IR of the composite bone outcome (29.3 vs. 41.4 per 1000 patient-years), with significant risk reductions for this outcome [HR, 0.69; 95\% confidence interval (CI), 0.58-0.83] and fragility fracture (HR, 0.59; 95\% CI, 0.44-0.78).

Conclusion: EFV + TDF/FTC is associated with a lower risk of adverse bone outcomes compared with other TDF-containing regimens in the VHA.

Funding: Bristol-Myers Squibb.

Keywords: Efavirenz; Fracture; Osteoporosis; Tenofovir disoproxil fumarate; Veterans

\section{INTRODUCTION}

Patients with HIV infection have higher rates of osteoporosis and fragility fractures than uninfected individuals, which are not completely explained by differences in traditional risk factors such as age and body mass index [1]. Randomized controlled trials and observational studies suggest that HIV infection itself and the initiation of some antiretroviral therapies (ART) may independently increase the risk of bone adverse outcomes [1-8]. For these reasons, the safety profiles of antiretroviral agents are subject to increased scrutiny in an effort to reduce treatment-related side effects in the aging HIV population.

Tenofovir disoproxil fumarate (TDF)/emtricitabine (FTC) has been the main nucleos(t)ide reverse transcriptase inhibitor backbone of combination ART for more than a decade; TDF/ FTC continues to be included in four of the six recommended regimens for treatment-naïve patients with HIV infection in the current Department of Health and Human Services guidelines [9]. TDF has been noted to increase the risk of fragility fractures $[7,10]$. In a Veterans Health Administration (VHA) population, cumulative exposure to TDF was independently predictive of fragility fracture [11]; however, no evaluation of TDF-associated fracture risk based on concomitantly administered ART was undertaken. Very few epidemiologic studies in US populations have compared risks of bone adverse outcomes with TDF across differing TDF-containing antiretroviral regimens [12].

ART initiation is associated with increases in bone turnover and modest decreases in bone mineral density (BMD) [13], which are greater with TDF-containing regimens. BMD loss with TDF is amplified when coadministered with boosted protease inhibitors (PIs), but effects are less certain when coadministered with other antiretroviral agents [7, 10, 14-25]. For example, in the AIDS Clinical Trials Group (ACTG) A5224s substudy of ACTG A5202, spine BMD loss was significantly greater in those receiving ritonavir-boosted atazanavir (ATV) + TDF/FTC $(-3.1 \%)$ than in those receiving efavirenz $($ EFV $)+$ TDF/FTC $(-1.7 \% ; P=0.035)$ [7]. However, the clinical relevance of these observations (increased fracture risk) remains uncertain.

Regarding fracture risk, available data from randomized controlled trials are limited by the small sample sizes and short observation periods of individual trials and the lack of specific reporting of fragility fractures [26]. Thus, despite differences in BMD across regimens in ACTG A5224s, fractures (all traumatic) were uncommon, and rates were similar across regimens [7]. In contrast, one recent large cohort study of commercial claims data showed lower fracture incidence rates (IRs) for EFV + TDF/FTC compared with elvitegravir/cobicistat (EVG/c)/ TDF/FTC, rilpivirine (RPV) + TDF/FTC, and the overall HIV population in the database [12].

The lower BMD loss and fracture risk with EFV-containing TDF/FTC regimens compared with other third agents combined with TDF/ FTC may relate to differences in drug-drug and drug-food interactions across these regimens. Tenofovir plasma concentrations are increased by $22-37 \%$ when TDF is taken with PIs [27, 28], cobicistat [29], or RPV [30]. The bioavailability of tenofovir is also increased by up to $40 \%$ with concomitant food intake $[28,31]$. Certain TDF regimens, including RPV, EVG/c, and boosted PIs, are taken with food [32-34]. Conversely, 
$\mathrm{EFV}+\mathrm{TDF} / \mathrm{FTC}$ is taken in the fasted state, and no clinically relevant drug-drug interactions have been reported between TDF and either EFV or FTC. Therefore, we hypothesized that $\mathrm{EFV}+\mathrm{TDF} / \mathrm{FTC}$ could result in a lower incidence of bone adverse outcomes compared with these other TDF-containing regimens.

The purpose of this analysis was to compare the incidence of bone adverse outcomes among treatment-naïve HIV-infected US veterans without bone disease who initiated different TDF-containing regimens.

\section{METHODS}

\section{Study Design and Data Sets}

Using a national cohort of US veterans, we conducted a population-based historical cohort study using VHA databases containing clinical, pharmacy, and administrative data from more than 150 VHA hospitals and 850 outpatient clinics nationwide [35]. We obtained demographic, laboratory, diagnosis, and utilization data from the Veterans Affairs (VA) Corporate Data Warehouse (CDW), including medical SAS data sets for in- and outpatient encounter data, CDW's raw pharmacy data, and Decision Support Systems and CDW's laboratory data. To conduct the analyses, data sets were housed in the VA Informatics and Computing Infrastructure environment, which enables access to data and tools for reporting and analysis in a secure workspace to ensure veterans' privacy and data security. This article does not contain any studies with human participants or animals performed by any of the authors. The University of Utah Institutional Review Board and the Salt Lake City VA Health Care System Office of Research and Development approved this study.

\section{Patients}

The cohort included all HIV-infected antiretroviral-naïve veterans without bone disease who initiated TDF/FTC plus a third antiretroviral agent of interest (see Exposures section) during the period 2003-2015. A validated algorithm
[36] with a sensitivity of $86 \%$ and positive predictive value of $87 \%$ [37] was used to exclude patients with evidence of prior ART received outside of the VHA, which included the following criteria: exposure to any antiretroviral agents during a 1-year period before the index date (the pre-index period), patients whose index ART regimen was a "salvage" regimen (i.e., composed of both a PI and an NNRTI or composed of 5 or more agents), and patients whose HIV RNA levels before the index date were low enough $(<500$ copies $/ \mathrm{ml})$ to suggest prior antiretroviral exposure. Veterans were identified for inclusion by an available index date for the first pharmacy fill for one of the third agents of interest and if they fulfilled the following criteria at the index date: (1) aged $\geq 18$ years; (2) at least 6 months of pre-index date VHA activity including in- or outpatient services; (3) no evidence of prior treatment including fills for antiretrovirals in the 6 months before the index date; (4) no evidence of prior bone disease (defined as diagnosis of osteoporosis/osteopenia by International Classification of Disease diagnosis codes, Current Procedural Terminology codes, or classification by bone mineral density test result). Codes used to identify patients with HIV infection are shown in Supplemental Digital Content, Table 1.

\section{Exposures}

Exposures of interest included TDF/FTC (either as a fixed-dose combination or as separate agents) plus one of the following agents: EFV, $\mathrm{EVG} / \mathrm{c}$, RPV, or any one of three ritonavirboosted PIs (i.e., ATV, lopinavir, or darunavir). For regimens with separate dosage forms, the third agent must have overlapped with the backbone within 30 days. For boosted or enhanced regimens (EVG/c and RTV-boosted PIs), the third agent must have also overlapped with the booster/enhancer for the patient to be classified as taking the regimen. Discontinuation of the regimen was defined as having a gap of at least 30 days for either the third agent or the backbone; patients who discontinued their regimen were censored on the first day of the 
Table 1 Unadjusted baseline characteristics among HIV-infected veterans receiving initial ART with differing TDF/FTCcontaining regimens

\begin{tabular}{|c|c|c|c|c|c|}
\hline & \multicolumn{5}{|c|}{ Initial ART Containing TDF/FTC Plus } \\
\hline & $\begin{array}{l}\text { EFV } \\
(n=4137)\end{array}$ & $\begin{array}{l}\text { Non-EFV } \\
(n=3024)\end{array}$ & $\begin{array}{l}\text { EVG/c } \\
(n=232)\end{array}$ & $\begin{array}{l}\text { RPV } \\
(n=171)\end{array}$ & $\begin{array}{l}\text { RTV-boosted } \\
\text { PI }(n=2621)\end{array}$ \\
\hline \multicolumn{6}{|l|}{ Demographics and physical } \\
\hline Age, years, mean $\pm S D$ & $50 \pm 10$ & $49 \pm 9.8$ & $49 \pm 13$ & $47 \pm 13$ & $50 \pm 9.3$ \\
\hline Male & $4002(96.7)$ & $2903(96.0)$ & $221(95.3)$ & $161(94.2)$ & $2521(96.2)$ \\
\hline Married & $337(8.1)$ & $221(7.3)$ & $26(11.2)$ & $20(11.7)$ & $175(6.7)$ \\
\hline \multicolumn{6}{|l|}{ Race } \\
\hline White & $1240(30.0)$ & $911(30.1)$ & $73(31.5)$ & $48(28.1)$ & $790(30.1)$ \\
\hline Black & $2492(60.2)$ & $1762(58.3)$ & $124(53.4)$ & $104(60.8)$ & $1534(58.5)$ \\
\hline Hispanic & $260(6.3)$ & $207(6.8)$ & $19(8.2)$ & $10(5.8)$ & $178(6.8)$ \\
\hline Asian & $34(0.8)$ & $28(0.9)$ & $5(2.2)$ & $2(1.2)$ & $21(0.8)$ \\
\hline Other & $22(0.5)$ & $28(0.9)$ & $2(0.9)$ & $2(1.2)$ & $24(0.9)$ \\
\hline Missing & $89(2.2)$ & $88(2.9)$ & $9(3.9)$ & $5(2.9)$ & $74(2.8)$ \\
\hline $\mathrm{BMI}\left(\mathrm{kg} / \mathrm{m}^{2}\right.$, mean $\left.\pm \mathrm{SD}\right)$ & $26 \pm 5.0$ & $25 \pm 5.1$ & $26 \pm 5.6$ & $27 \pm 5.3$ & $25 \pm 5.1$ \\
\hline \multicolumn{6}{|l|}{ Pre-index ${ }^{\mathrm{b} \dagger}$ prognostic indices } \\
\hline \multicolumn{6}{|l|}{$\mathrm{CD} 4^{+}$count, cells $/ \mathrm{mm}^{3}$} \\
\hline$<200$ & $1286(31.1)$ & $1103(36.5)$ & $60(25.9)$ & $23(13.5)$ & $1020(38.9)$ \\
\hline $200-299$ & $698(16.9)$ & $477(15.8)$ & $33(14.2)$ & $30(17.5)$ & $414(15.8)$ \\
\hline $300-399$ & $634(15.3)$ & $342(11.3)$ & $34(14.7)$ & $11(6.4)$ & $297(11.3)$ \\
\hline $400-499$ & $401(9.7)$ & $222(7.3)$ & $24(10.3)$ & $33(19.3)$ & $165(6.3)$ \\
\hline$\geq 500$ & $588(14.2)$ & $426(14.1)$ & $61(26.3)$ & $63(36.8)$ & $302(11.5)$ \\
\hline Missing & $530(12.8)$ & $454(15.0)$ & $20(8.6)$ & $11(6.4)$ & $423(16.1)$ \\
\hline \multicolumn{6}{|l|}{ HIV viral load, copies/ml } \\
\hline$<10,000$ & $1184(28.6)$ & $950(31.4)$ & $65(28.0)$ & $68(39.8)$ & $817(31.2)$ \\
\hline $10,000-100,000$ & $1434(34.7)$ & $940(31.1)$ & $78(33.6)$ & $67(39.2)$ & $795(30.3)$ \\
\hline$>100,000$ & $913(22.1)$ & $686(22.7)$ & $61(26.3)$ & $15(8.8)$ & $610(23.3)$ \\
\hline Missing & $606(14.6)$ & $448(14.8)$ & $28(12.1)$ & $21(12.3)$ & $399(15.2)$ \\
\hline \multicolumn{6}{|l|}{ Pre-index ${ }^{\mathrm{b}}$ renal function } \\
\hline \multicolumn{6}{|l|}{$\mathrm{eGFR}, \mathrm{ml} / \mathrm{min} / 1.73 \mathrm{~m}^{2}$} \\
\hline$\geq 90$ & $2193(53.0)$ & $1552(51.3)$ & $143(61.6)$ & $108(63.2)$ & $1301(49.6)$ \\
\hline $60-89$ & $1360(32.9)$ & $985(32.6)$ & $73(31.5)$ & $54(31.6)$ & $858(32.7)$ \\
\hline $30-59$ & $164(4.0)$ & $123(4.1)$ & $3(1.3)$ & $4(2.3)$ & $116(4.4)$ \\
\hline $15-29$ & $5(0.1)$ & $7(0.2)$ & $0(0.0)$ & $0(0.0)$ & $7(0.3)$ \\
\hline$<15$ & $39(0.9)$ & $53(1.8)$ & $0(0.0)$ & $0(0.0)$ & $53(2.0)$ \\
\hline Missing & $376(9.1)$ & $304(10.1)$ & $13(5.6)$ & $5(2.9)$ & $286(10.9)$ \\
\hline
\end{tabular}


Table 1 continued

\section{Initial ART Containing TDF/FTC Plus}

\begin{tabular}{lllll}
\hline EFV & Non-EFV & EVG/c & RPV & RTV-boosted \\
$(n=4137)$ & $(n=3024)$ & $(n=232)$ & $(n=171)$ & PI $(n=2621)$
\end{tabular}

Pre-index ${ }^{\mathrm{b}}$ comorbid diagnoses

\begin{tabular}{|c|c|c|c|c|c|}
\hline $\mathrm{CAD} / \mathrm{CVD}$ & $447(10.8)$ & $280(9.3)$ & $28(12.1)$ & $13(7.6)$ & $239(9.1)$ \\
\hline Heart failure & $132(3.2)$ & $62(2.1)$ & $5(2.2)$ & $2(1.2)$ & $55(2.1)$ \\
\hline Dyslipidemia & $659(15.9)$ & $412(13.6)$ & $50(21.6)$ & $29(17.0)$ & $333(12.7)$ \\
\hline Hypertension & $1521(36.8)$ & $910(30.1)$ & $83(35.8)$ & $64(37.4)$ & $763(29.1)$ \\
\hline Diabetes mellitus & $561(13.6)$ & $358(11.8)$ & $30(12.9)$ & $19(11.1)$ & $309(11.8)$ \\
\hline Chronic kidney disease $^{c}$ & $281(6.8)$ & $169(5.6)$ & $16(6.9)$ & $9(5.3)$ & $144(5.5)$ \\
\hline End-stage renal disease ${ }^{\mathrm{d}}$ & $15(0.4)$ & $12(0.4)$ & $1(0.4)$ & $0(0.0)$ & $11(0.4)$ \\
\hline Fracture & $30(0.7)$ & $20(0.7)$ & $4(1.7)$ & $1(0.6)$ & $15(0.6)$ \\
\hline Viral hepatitis & $1122(27.1)$ & $902(29.8)$ & $59(25.4)$ & $42(24.6)$ & $801(30.6)$ \\
\hline Tuberculosis & $52(1.3)$ & $46(1.5)$ & $1(0.4)$ & $2(1.2)$ & $43(1.6)$ \\
\hline Psychiatric disorder & $1495(36.1)$ & $1384(45.8)$ & $119(51.3)$ & $104(60.8)$ & $1161(44.3)$ \\
\hline Depression & $962(23.3)$ & $878(29.0)$ & $83(35.8)$ & $68(39.8)$ & $727(27.7)$ \\
\hline Schizophrenia & $140(3.4)$ & $194(6.4)$ & $12(5.2)$ & $9(5.3)$ & $173(6.6)$ \\
\hline Bipolar disorder & $635(15.3)$ & $648(21.4)$ & $61(26.3)$ & $63(36.8)$ & $524(20.0)$ \\
\hline Psychosis & $231(5.6)$ & $308(10.2)$ & $26(11.2)$ & $18(10.5)$ & $264(10.1)$ \\
\hline Posttraumatic stress disorder & $366(8.8)$ & $379(12.5)$ & $39(16.8)$ & $52(30.4)$ & $288(11.0)$ \\
\hline Tobacco use $\mathrm{e}^{\mathrm{e}}$ & $1279(30.9)$ & $918(30.4)$ & $70(30.2)$ & $77(45.0)$ & $771(29.4)$ \\
\hline Alcohol abuse & $946(22.9)$ & $725(24.0)$ & $59(25.4)$ & $51(29.8)$ & $615(23.5)$ \\
\hline \multicolumn{6}{|l|}{ Medications } \\
\hline Methadone & $49(1.2)$ & $70(2.3)$ & $2(0.9)$ & $2(1.2)$ & $66(2.5)$ \\
\hline Proton pump inhibitors & $1179(28.5)$ & $713(23.6)$ & $56(24.1)$ & $23(13.5)$ & $634(24.2)$ \\
\hline Bisphosphonates & $8(0.2)$ & $9(0.3)$ & $0(0.0)$ & $0(0.0)$ & $9(0.3)$ \\
\hline Testosterone & $66(1.6)$ & $51(1.7)$ & $2(0.9)$ & $3(1.8)$ & $46(1.8)$ \\
\hline
\end{tabular}

Data are $n(\%)$ unless otherwise indicated

a Non-EFV includes the EVG/c, RPV, and RTV-boosted PI groups

b Pre-index comorbidities and clinical characteristics were identified in the 6-12-month pre-index period

${ }^{c}$ Defined as either a chronic kidney disease diagnosis or two consecutive measures of eGFR $<60 \mathrm{ml} / \mathrm{min} / 1.73 \mathrm{~m}^{2}$ occurring at least 30 days apart

${ }^{d}$ Defined as either a diagnosis of end-stage renal disease, kidney transplant, or dialysis

e Includes abuse, dependence, rehabilitation, and toxicity related to tobacco

$A R T$ antiretroviral therapy, $B M I$ body mass index, $C A D$ coronary artery disease, $C V D$ cerebrovascular disease, $E F V$ efavirenz, $e G F R$ estimated glomerular filtration rate, $E V G / c$ elvitegravir/cobicistat, $F T C$ emtricitabine, $P I$ protease inhibitor (atazanavir, darunavir, or lopinavir), $R P V$ rilpivirine, $R T V$ ritonavir, $S D$ standard deviation, $T D F$ tenofovir disoproxil fumarate 
first 30-day gap following the end of the prior days' supply received by the patient.

\section{Outcomes}

The primary composite outcome was a bone adverse event defined as a diagnosis of osteoporosis; a BMD T-score in the osteoporotic or osteopenic ranges for the femoral neck, total spine, distal radius, or total hip; or a diagnosis or procedure code for likely fragility fracture (any hip, wrist/forearm, or spine fracture). BMD T-scores were extracted from patient radiology dual-energy X-ray absorptiometry (DEXA) reports and clinical notes using a previously developed natural language processing tool, with accuracy in the range of 90.4-92.8\% $[38,39]$. All codes used to identify outcomes of interest are provided in Supplemental digital content, Table 2.

\section{Covariates}

To control for confounding and selection bias, we measured baseline covariates that were selected on the basis of potential associations with treatment and/or outcomes, as found in published literature and based on prior clinical knowledge of ART and HIV. These included baseline demographics, baseline HIV laboratory measures, baseline BMD measures and related diagnoses, lifestyle exposures, other comorbidities, medication exposures, and calendar year of the index regimen. All covariates were identified over a 12-month look-back period preceding the index date. Specific definitions for all covariates are provided in the Supplemental digital content, Table 3.

\section{Statistical Analysis}

We calculated baseline characteristics overall and by treatment group and used standardized mean differences (SMDs) to compare differences between groups, with SMDs outside the bounds of \pm 0.1 indicating meaningful differences [40]. We calculated crude IRs of bone adverse outcomes per 1000 patient-years of exposure and associated exact 95\% confidence intervals (CIs) in the unweighted cohort assuming a Poisson distribution. To control for confounding by indication and selection bias, we used inverse probability of treatment weighting (IPTW) for each patient [41]. Weighted Cox proportional hazards regression models were used to estimate adjusted hazard ratios (HRs) for bone outcomes associated with EFV + TDF/FTC compared with $\mathrm{EVG} / \mathrm{c}+\mathrm{TDF} / \mathrm{FTC}$, RPV + TDF/FTC, ritonavirboosted PIs + TDF/FTC, and all non-EFV regimens combined. To reduce risk of bias, model variability, and unreliable CIs, we excluded any group with $<5$ events from analysis comparisons. We used IPTW in the primary analysis; however, if covariate balance was not achieved with IPTW, we conducted a sensitivity analysis using matching weights, which are less sensitive to residual and unmeasured confounding. All analyses were done in SAS version 9.2 (SAS Institute, Cary, NC).

\section{RESULTS}

\section{Patient Characteristics}

A total of 7161 patients met all eligibility criteria (Fig. 1). Of these, 4137 initiated EFV and 3024 initiated non-EFV regimens, including 232 with EVG/c, 171 with RPV, and 2621 with ritonavir-boosted PIs. At baseline, 26 (0.4\%) had received a bisphosphonate (Table 1). For all unadjusted comparisons, psychiatric disorders were overrepresented in those receiving nonEFV-containing regimens versus EFV-containing regimens (Fig. 2 and Supplemental digital content Table 4). After IPTW, covariate balance was achieved among all variables for EFV versus all non-EFV combined (Fig. 2), versus EVG/c (Supplemental digital content, Fig. 1A), and versus ritonavir-boosted PIs (Supplemental digital content, Fig. 1B). For EFV versus RPV, the covariate balance was achieved for $83.0 \%$ of variables (Supplemental digital content, Fig. 1C). Mean (standard deviation) follow-up times were 13.0 (19.2) months overall and 15.0 (21.2), 7.6 (7.6), 10.0 (10.5), and 10.5 (16.7) months for EFV, EVG/c, RPV, and ritonavirboosted PIs, respectively. Corresponding median (interquartile range; min-max) values were 


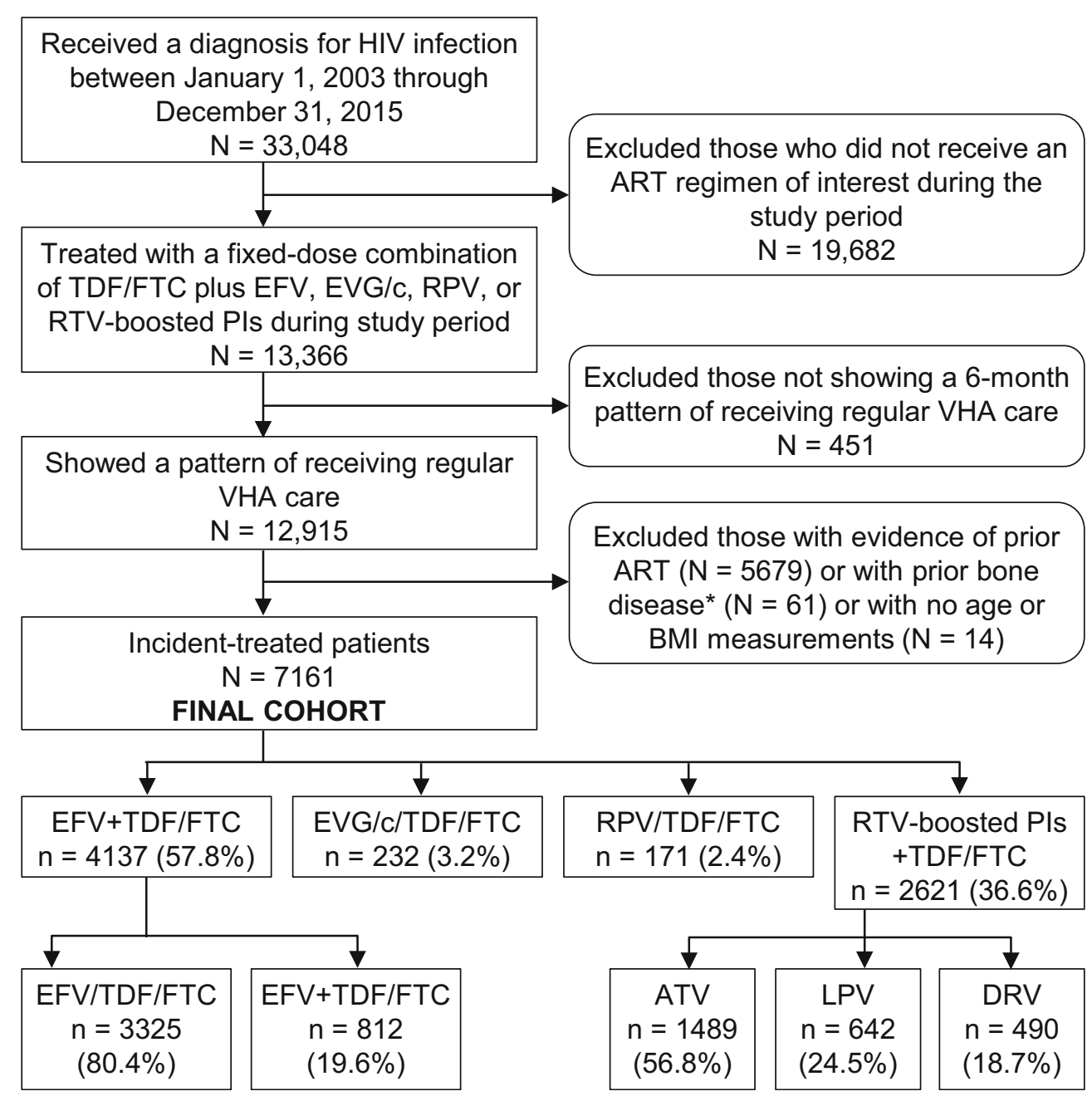

Fig. 1 Patient selection according to eligibility criteria. $A R T$ antiretroviral therapy, $A T V$ atazanavir, $B M I$ body mass index, $D R V$ darunavir, $E F V$ efavirenz, $E V G / c$ elvitegravir/cobicistat, FTC emtricitabine, $L P V$ lopinavir, $P I$ protease inhibitor (atazanavir, darunavir, or lopinavir), $R P V$ rilpivirine, $R T V$ ritonavir, $T D F$ tenofovir disoproxil

\section{$5.0 \quad(2.0-14.2 ; \quad 0.1-127.7), \quad 5.7 \quad(2.1-17.9 ;$ $0.1-110.3), \quad 4.6 \quad(2.1-10.4 ; \quad 0.2-34.6), \quad 5.9$ $(2.8-13.4 ; \quad 0.5-48.3), \quad$ and $4.2 \quad(1.8-10.7$;} 0.1-127.7) months.

\section{Incidence and Risk of Any Bone Outcome}

The unadjusted (crude) IRs and adjusted HRs for all comparisons are summarized in Fig. 3. The crude IR of the composite bone outcome was lower in the EFV group than in the EVG/c, RPV, and ritonavir-boosted PI groups. In adjusted fumarate, $V H A$ Veterans Health Administration. ${ }^{*}$ Bone disease defined as diagnosis of osteoporosis/osteopenia by international classification of disease diagnosis codes, current procedural terminology codes, or classification by bone mineral density test result

analyses, EFV was associated with a statistically significant $31 \%$ lower risk of the composite bone outcome than non-EFV groups combined, 25\% lower than RPV, and 30\% lower than the ritonavir-boosted PI group.

\section{Incidence and Risk of Osteoporosis or Osteopenia}

The crude IR for osteoporosis was lower for EFV compared with all other regimens. In adjusted analyses, EFV was associated with a statistically 


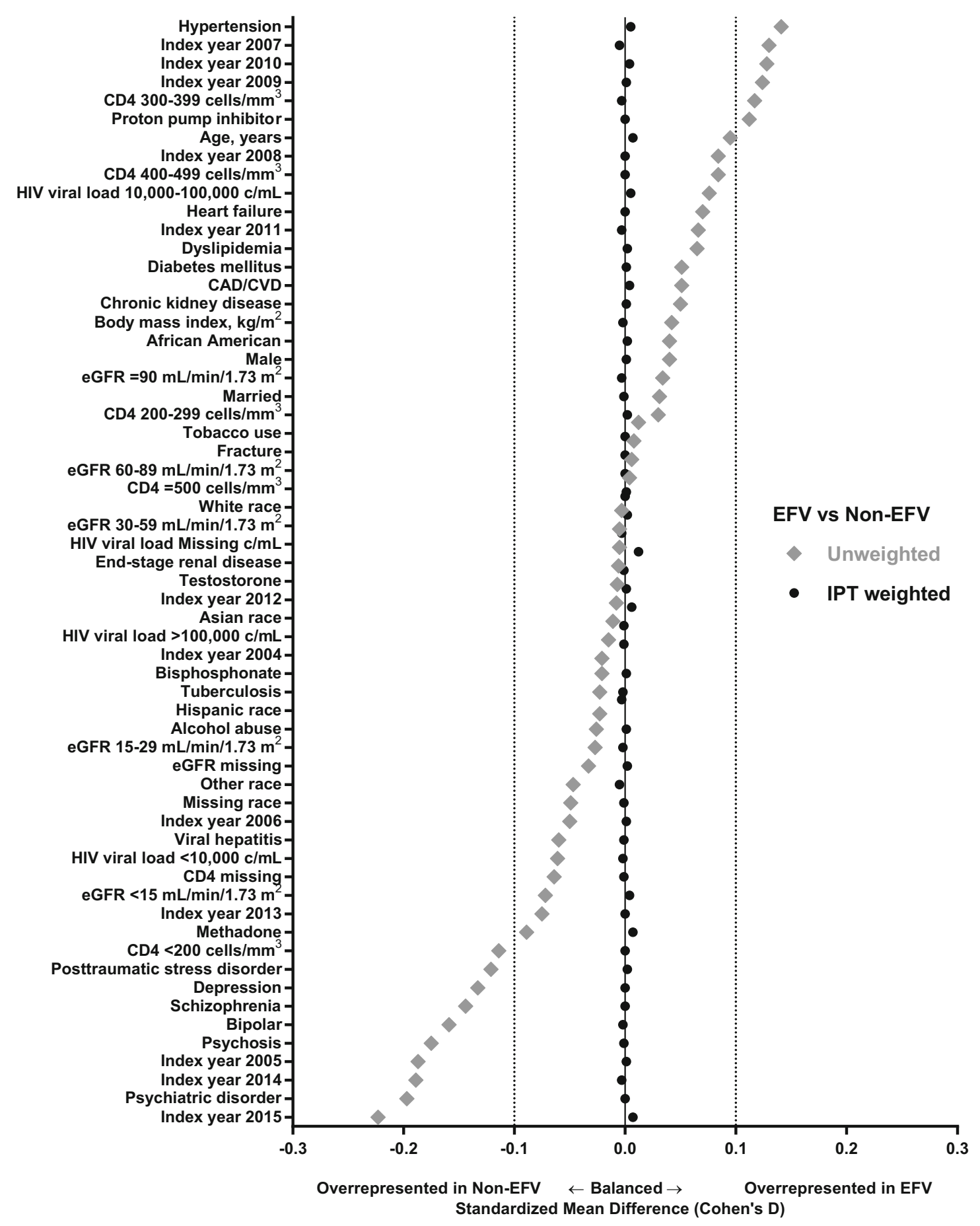

Fig. 2 Baseline demographics: verification that IPT weighting achieves baseline covariate balance between TDF/FTC-containing regimens with EFV versus all nonEFV. $C A D / C V D$ coronary artery disease/cerebrovascular disease, $C K D$ chronic kidney disease, $E F V$ efavirenz, $e G F R$ estimated glomerular filtration rate, $F T C$ emtricitabine, $I P T$ inverse probability of treatment, TDF tenofovir disoproxil fumarate 
significant $36 \%$ lower risk of osteoporosis than non-EFV groups combined, 53\% lower than $\mathrm{EVG} / \mathrm{c}$, and $35 \%$ lower than the ritonavirboosted PI group. Too few events were observed with RPV to make comparisons. The crude IR of osteopenia was lower for EFV compared with $\mathrm{EVG} / \mathrm{c}$, but for all other comparisons IRs were similar. In adjusted analyses, the risk of osteopenia was similar between EFV and the ritonavir-boosted PI group (too few events were observed to report comparisons for EVG/c or RPV). When combining osteoporosis and osteopenia events, EFV was associated with a $21 \%$ lower risk compared with the ritonavirboosted PI group. Frequencies of available preand post-index DEXA scans as well as baseline characteristics by DEXA scan status are presented in the Supplemental digital content, Tables 6 and 7, respectively.

\section{Incidence and Risk of Fracture}

The crude IR of fragility fractures was lower for EFV compared with EVG/c, RPV, and ritonavirboosted PIs. In adjusted analyses, risk differences were statistically significant for EFV versus all non-EFV combined (41\% lower), RPV (57\% lower), and ritonavir-boosted PIs (40\% lower).

Of the individual fracture outcomes, EFV had a lower incidence compared with each non-EFV regimen for all fracture sites in all comparisons except for hip fractures with ritonavir-boosted PIs, which had too few events to make comparisons. In adjusted analyses, this corresponded to statistically significant reduced risks for EFV compared with all non-EFV regimens combined for vertebral fracture (51\% lower) and wrist/forearm fracture (60\% lower). Significantly lower risks were also observed versus ritonavir-boosted PIs for vertebral fracture (48\% lower) and wrist/forearm fracture (60\% lower). Too few events were observed for individual fracture outcomes to make comparisons with $\mathrm{EVG} / \mathrm{c}$ or RPV.

For a summary of event numbers, time to events and follow-up times for all bone adverse outcomes, see Supplemental digital content, Table 5.

\section{Sensitivity Analysis}

In matching weight analyses, the risks of any bone outcome or fracture for RPV versus EFV showed a similar magnitude, suggesting the IPTW findings were robust regarding residual confounding present for $7 / 47$ (14.9\%) of variable comparisons for EFV versus RPV.

\section{DISCUSSION}

EFV + TDF/FTC was generally associated with lower risks of bone adverse outcomes compared with TDF-containing regimens with EVG/c, $\mathrm{RPV}$, or ritonavir-boosted PIs in the VHA cohort over an average follow-up time of 13.0 months. These findings suggest that patients with HIV infection receiving EFV + TDF/FTC, which has no known drug-drug interactions with TDF or FTC, or drug-food interactions, may be at lower overall risk of bone adverse outcomes compared with those receiving TDF-containing regimens with EVG/c, RPV, or ritonavir-boosted PIs.

This study is the first to identify an association between EFV + TDF/FTC use among veterans and a reduced risk of bone adverse outcomes versus other TDF-containing regimens. These findings confirm those of Nkhoma et al. [12], who conducted a large claims database analysis of bone fractures associated with TDF/FTC and various third agents (although adjusted analyses were not possible because of insufficient numbers of fracture events). Nkhoma et al. [12] found a lower fracture IR with EFV + TDF/FTC (3.4 per 1000 patientyears) compared with RPV + TDF/FTC (3.6 per 1000 patient-years) or with EVG/c + TDF/FTC (4.4 per 1000 patient-years). The IRs in this study followed the same general trend as in Nkhoma et al. [12], but were higher in magnitude, possibly because the current study was conducted in a higher-risk population in a relatively closed, integrated care network.

Cobicistat, rilpivirine, and ritonavir increase serum creatinine and decrease the estimated glomerular filtration rate (GFR) via inhibition of renal tubular transporters $[42,43]$, but this does not appear to affect actual GFR as measured by iohexol clearance [44]. Therefore, the bone 
Crude Incidence $(95 \% \mathrm{Cl})$

per $1000 \mathrm{PY}$

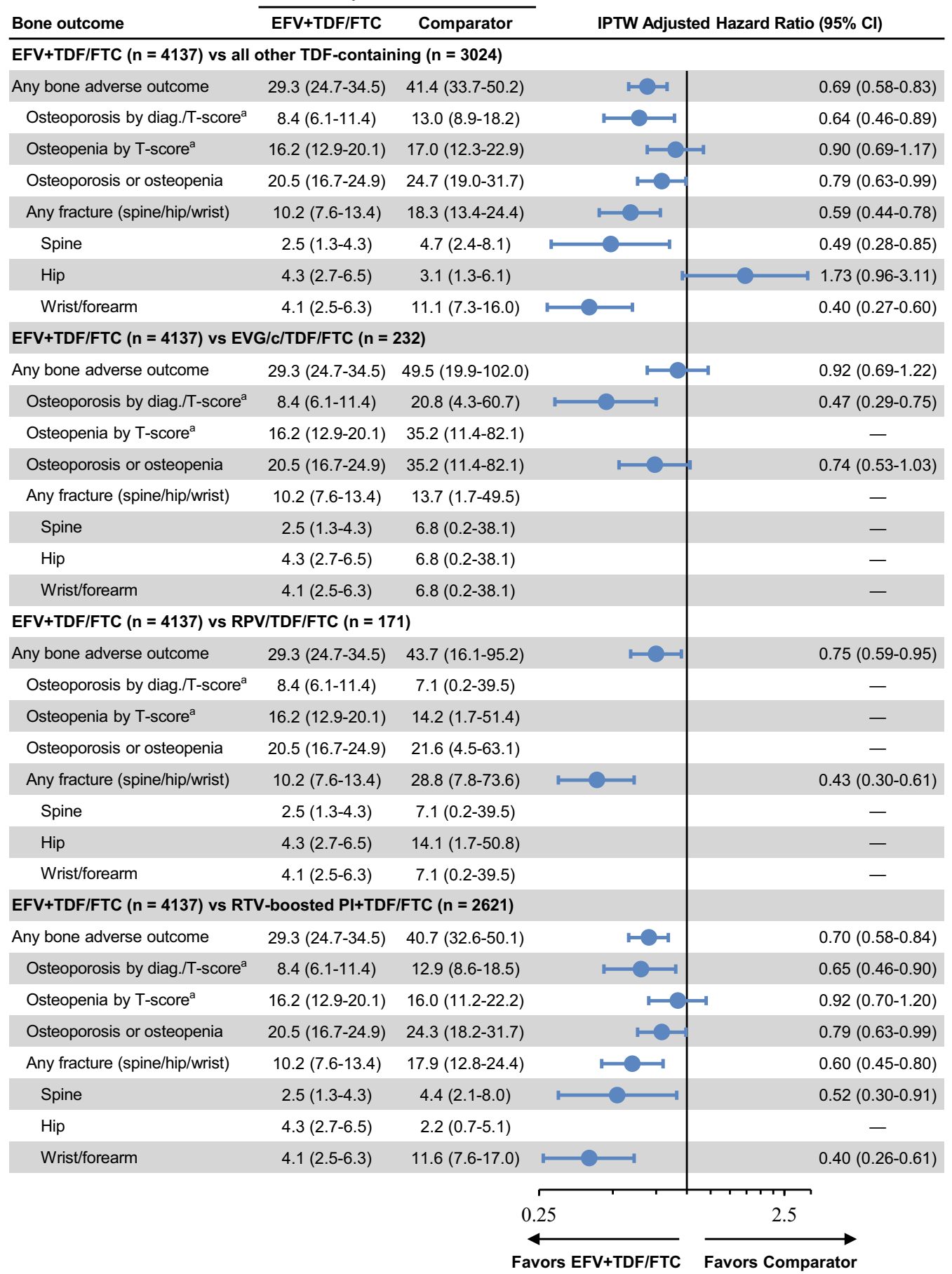

Fig. 3 Unadjusted incidence rates and IPTW adjusted hazard ratios of bone adverse outcomes among patients treated with different TDF/FTC-containing regimens with at least five events per group. $C I$ confidence interval, $C V D$ cerebrovascular disease, diag. diagnosis, $E F V$ efavirenz, $E V G / c$ elvitegravir/cobicistat, FTC emtricitabine,
IPTW inverse probability of treatment weighting, $P I$ protease inhibitor (atazanavir, darunavir, or lopinavir), $P Y$ patient-years, $R P V$ rilpivirine, $R T V$ ritonavir, $T D F$ tenofovir disoproxil fumarate, - insufficient events to calculate hazard ratio. ${ }^{a}$ Measured at the femoral neck, total spine, distal radius, or total hip 
effects we observed were more likely due to relative differences in tenofovir exposure across the evaluated regimens. Both RPV/FTC/TDF and EVG/COBI/FTC/TDF are recommended to be administered with food, which increases tenofovir exposure compared with fasting administration [33, 34]. In addition, both protease inhibitors (including RTV) [45] and cobicistat [46] increase tenofovir exposure by inhibition of intestinal P-gp-mediated efflux of TDF. Taken together, these effects on tenofovir exposure may have contributed to the increased risk of adverse bone outcomes that we observed with EVG/c/FTC/TDF, RPV/TDF/FTC, and RTV-boosted PIs in this analysis.

For patients with a high risk of bone adverse outcomes, the use of the novel formulation tenofovir alafenamide (TAF) or abacavir has been associated with lower BMD losses at the time of therapy initiation compared with TDF [9]. TAF, a prodrug of tenofovir, is associated with a 91\% lower plasma tenofovir concentration than that following TDF administration while maintaining higher intracellular tenofovir concentrations in peripheral blood mononuclear cells for HIV suppression [47]. Biomarkers of bone turnover appear to be less affected by TAF-containing regimens compared with TDF-containing regimens. TAF is now recommended in HIV treatment guidelines [9], and its use is being preferred to TDF by HIV-care providers concerned about bone toxicity of TDF in the aging HIV population.

Given the ongoing widespread use of EFV + TDF/FTC and the absence of an EFV coformulation with TAF, the results of the current study may reassure physicians and their patients about the bone safety of this combination. Moreover, where alternatives to TDF are limited (such as in resource-limited settings), or where use of generic EFV or TDF as a cost reduction strategy is available, or where $\mathrm{EFV}+\mathrm{TDF} / \mathrm{FTC}$ is available through the Medicines Patent Pool, the choice of a third agent remains critical to long-term safety. The reduced risk of bone adverse outcomes for $\mathrm{EFV}+\mathrm{TDF} / \mathrm{FTC}$ found in this study is of high relevance, especially in resource-limited settings where the cost effectiveness of the fixed-dose combination has achieved widespread use [48].
However, while it appears that EFV + TDF/FTC has a lower risk for bone adverse outcomes than PIs and other boosted regimens in combination with TDF/FTC, our study is not generalizable to the use of unboosted integrase inhibitors, for which additional research is needed.

Other options to counter antiretroviral-associated BMD loss include vitamin D/calcium and/ or zoledronic acid supplementation. Vitamin $\mathrm{D} /$ calcium supplementation lessened BMD loss among patients receiving EFV + TDF/FTC over 48 weeks [21, 23], and single-dose zoledronic acid administered at the initiation of a PI-containing TDF/FTC regimen can prevent the initial BMD loss [49]. These options, together with careful antiretroviral choice, should be considered between patients and health care providers, taking into account the drug resistance profile, treatment history, other comorbidities, and the risk or tolerability of side effects.

Strengths and limitations of this study are those common to large epidemiologic studies. The main strengths of our study were its large sample size and detailed data from VHA data sets, as well as the use of a natural languageprocessing tool, which allowed us to extract BMD results from radiology and clinical notes. Our study also has limitations. The VHA population was more than 95\% male, which limits generalizability to female populations that could be affected differently by the predictors identified in our study. Follow-up times were short, which may have led to underascertainment of relevant bone adverse outcomes; however, statistically significant between-regimen differences for relevant bone adverse outcomes were noted despite these follow-up times. Moreover, other data have demonstrated that the incidence of fracture is highest during the first and second years after ART initiation, tailing off thereafter [50]; fracture risk among men with HIV infection is higher among older individuals [51] as was the case in this VA population. Thus, we consider that follow-up times in the current study were sufficient to detect differences in BMD and fracture risk. Although IPTW was successfully used to adjust for selection bias and measured confounders for all comparisons with the exception of EFV versus $\mathrm{RPV}$, the potential for unmeasured confounders 
and incomplete adjustment for measured confounders cannot be ruled out. For example, patients with a range of psychiatric disorders were less likely to be prescribed EFV-containing regimens across all comparisons (Fig. 2 and Supplemental digital content, Fig. 1), likely because of channeling bias consequent to the side effect profile of EFV in patients with severe psychiatric symptoms [52]. Various psychiatric disorders, such as schizophrenia and depression, and psychotropic medications are associated with BMD loss and an increased risk of fracture [53]. However, our method of controlling for confounding effectively balanced the observed differences in these measured characteristics. Specifically, the six psychiatric covariates examined were balanced after IPTW for the $\mathrm{EFV}$ versus EVG/c and EFV versus ritonavirboosted PI comparisons. For the EFV versus RPV comparison, only one remained imbalanced after IPTW, and this was by a small margin (SMD of 0.102). In addition, the more conservative matching weights analyses produced qualitatively similar results to those using IPTW, making it highly unlikely that channeling bias affected our results. Finally, as in any retrospective observational study, causal associations cannot be proven; thus, these findings require confirmation in further prospective studies.

\section{CONCLUSION}

In conclusion, EFV + TDF/FTC was generally associated with a lower incidence of bone adverse outcomes, including osteoporosis, any major fracture, vertebral fracture, and wrist/forearm fracture, compared with other TDF/FTC-containing regimens in the VHA. The third agent in antiretroviral regimens may have a significant effect on the risk of bone adverse events associated with TDF.

\section{ACKNOWLEDGMENTS}

Funding. This work was supported by Bristol-Myers Squibb by a grant to the University of
Utah. The article processing charges were also funded by Bristol-Myers Squibb.

Authorship. All authors had full access to all of the data in this study and take complete responsibility for the integrity of the data and accuracy of the data analysis. All named authors meet the International Committee of Medical Journal Editors (ICMJE) criteria for authorship for this manuscript, take responsibility for the integrity of the work as a whole, and have given final approval to the version to be published.

Author Contribution. Joanne LaFleur, Adam P. Bress, Joel Myers, Lisa Rosenblatt, Roger Bedimo, Pablo Tebas, Heather Nyman, and Stephen Esker contributed to the conception and design of the study. Joanne LaFleur, Adam P. Bress, Kristin Knippenberg, and Jacob Crook contributed to data collection. Joanne LaFleur, Adam P. Bress, Kristin Knippenberg, and Jacob Crook contributed to data analysis. Joanne LaFleur, Adam P. Bress, Joel Myers, Lisa Rosenblatt, Roger Bedimo, Pablo Tebas, Heather Nyman, and Stephen Esker contributed to data interpretation. All authors contributed to writing and editing the manuscript.

Editorial Assistance. Editorial Assistance was provided by Julian Martins of inScience Communications, Springer Healthcare, which was funded by Bristol-Myers Squibb.

Disclosures. This work was supported by Bristol-Myers Squibb by a grant to the University of Utah. Joanne LaFleur received some salary support from this grant and declares no intellectual property rights related to this research. Outside of the funded work, over the last 3 years, the following organizations provided research grants to the University of Utah and Joanne LaFleur worked on those projects and/or received salary or other types of support that were funded by those grants: Gilead Sciences, Inc., Anolinx LLC, Skaggs Foundation, and Agency for Healthcare Research and Quality. Adam P. Bress received some salary support from this grant and declares no intellectual property rights related to this research. Outside of the funded work, over the last 3 years, the following 
organizations provided research grants to the University of Utah, and Adam P. Bress worked on those projects and/or received salary or other types of support that were funded by those grants: Gilead Sciences, and National Heart, Lung, and Blood Institute (NHLBI). Joel Myers is an employee of, and owns stock in, Bristol-Myers Squibb. Lisa Rosenblatt is an employee of, and owns stock in, Bristol-Myers Squibb. Jacob Crook received some salary support from this grant and declares no intellectual property rights related to this research. Outside of the funded work, over the last 3 years, the following organizations provided research grants to the University of Utah and Jacob Crook worked on those projects and/or received salary or other types of support that were funded by those grants: Cubist, Gilead Sciences, Inc., Anolinx LLC, Skaggs Foundation, Agency for Healthcare Research and Quality, and Utah Department of Health. Kristin Knippenberg received some salary support from this grant and declares no intellectual property rights related to this research. Outside of the funded work, over the last 3 years, the following organizations provided research grants to the University of Utah and Kristin Knippenberg worked on those projects and/or received salary or other types of support that were funded by those grants: Gilead Sciences, Inc., Anolinx LLC, Skaggs Foundation, Agency for Healthcare Research and Quality, and Utah Department of Health. Roger Bedimo has received grants and research support awarded to the Veterans Affairs North Texas Healthcare System from Merck \& Co; he has served as a scientific advisor for Bristol-Myers Squibb, Merck \& Co, Inc., and Theratechnologies, Inc. Pablo Tebas has served as a scientific advisor to Merck \& Co, Inc., and is a member of an adjudication committee in a vaccine trial sponsored by Glaxo. Heather Nyman received some salary support and travel expenses from this grant and declares no intellectual property rights related to this research; she has also received consultancy honoraria from Otsuka and Fresenius. Stephen Esker is an employee of, and owns stock in, Bristol-Myers Squibb.

Compliance with Ethics Guidelines. This article does not contain any studies with human participants or animals performed by any of the authors. The University of Utah Institutional Review Board and the Salt Lake City VA Health Care System Office of Research and Development approved this study.

Data Availability. The data sets generated during and/or analyzed during the current study are not publicly available because of compliance with Veteran Healthcare Administration restrictions on data sharing.

Previous Presentation. These data were previously presented in poster form at Infectious Disease Week (IDWeek) October 26-30, 2016, New Orleans, LA.

Open Access. This article is distributed under the terms of the Creative Commons Attribution-NonCommercial 4.0 International License (http://creativecommons.org/licenses/ by-nc/4.0/), which permits any noncommercial use, distribution, and reproduction in any medium, provided you give appropriate credit to the original author(s) and the source, provide a link to the Creative Commons license, and indicate if changes were made.

\section{REFERENCES}

1. Cotter AG, Sabin CA, Simelane S, et al. Relative contribution of HIV infection, demographics and body mass index to bone mineral density. AIDS. 2014;28(14):2051-60.

2. Brown TT, Qaqish RB. Antiretroviral therapy and the prevalence of osteopenia and osteoporosis: a meta-analytic review. AIDS. 2006;20(17):2165-74.

3. Hileman CO, Labbato DE, Storer NJ, Tangpricha V, McComsey GA. Is bone loss linked to chronic inflammation in antiretroviral-naive HIV-infected adults? A 48-week matched cohort study. AIDS. 2014;28(12):1759-67.

4. Young B, Dao CN, Buchacz K, Baker R, Brooks JT. Investigators HIVOS. Increased rates of bone fracture among HIV-infected persons in the HIV Outpatient Study (HOPS) compared with the US general population, 2000-2006. Clin Infect Dis. 2011;52(8):1061-8. 
5. Yong MK, Elliott JH, Woolley IJ, Hoy JF. Low CD4 count is associated with an increased risk of fragility fracture in HIV-infected patients. J Acquir Immune Defic Syndr. 2011;57(3):205-10.

6. Grund B, Peng G, Gibert CL, et al. Continuous antiretroviral therapy decreases bone mineral density. AIDS. 2009;23(12):1519-29.

7. McComsey GA, Kitch D, Daar ES, et al. Bone mineral density and fractures in antiretroviral-naive persons randomized to receive abacavir-lamivudine or tenofovir disoproxil fumarate-emtricitabine along with efavirenz or atazanavir-ritonavir: Aids Clinical Trials Group A5224s, a substudy of ACTG A5202. J Infect Dis. 2011;203(12):1791-801.

8. van Vonderen MG, Lips P, van Agtmael MA, et al. First line zidovudine/lamivudine/lopinavir/ritonavir leads to greater bone loss compared to nevirapine/lopinavir/ritonavir. AIDS. 2009;23(11):1367-76.

9. Panel on Antiretroviral Guidelines for Adults and Adolescents. Guidelines for the use of antiretroviral agents in HIV-1-infected adults and adolescents. https://aidsinfo.nih.gov/guidelines/html/1/adultand-adolescent-arv-guidelines/11/what-to-start. Accessed 27 Feb 2017.

10. Stellbrink HJ, Orkin C, Arribas JR, et al. Comparison of changes in bone density and turnover with abacavir-lamivudine versus tenofovir-emtricitabine in HIV-infected adults: 48-week results from the ASSERT study. Clin Infect Dis. 2010;51(8):963-72.

11. Bedimo R, Maalouf NM, Zhang S, Drechsler H, Tebas P. Osteoporotic fracture risk associated with cumulative exposure to tenofovir and other antiretroviral agents. AIDS. 2012;26(7):825-31.

12. Nkhoma ET, Rosenblatt L, Myers J, Villasis-Keever A, Coumbis J. Real-world assessment of renal and bone safety among patients with hiv infection exposed to tenofovir disoproxil fumarate-containing singletablet regimens. PLoS ONE. 2016;11(12):e0166982.

13. Cazanave C, Dupon M, Lavignolle-Aurillac V, et al. Reduced bone mineral density in HIV-infected patients: prevalence and associated factors. AIDS. 2008;22(3):395-402.

14. Behrens G, Rijnders B, Nelson M, et al. Rilpivirine versus efavirenz with emtricitabine/tenofovir disoproxil fumarate in treatment-naive HIV-1-infected patients with HIV-1 RNA $\leq 100,000$ copies/mL: week 96 pooled ECHO/THRIVE subanalysis. AIDS Patient Care STDS. 2014;28(4):168-75.

15. Daar ES, Tierney C, Fischl MA, et al. Atazanavir plus ritonavir or efavirenz as part of a 3-drug regimen for initial treatment of HIV-1. Ann Intern Med. 2011;154(7):445-56.
16. Erlandson KM, Kitch D, Tierney C, et al. Weight and lean body mass change with antiretroviral initiation and impact on bone mineral density. AIDS. 2013;27(13):2069-79.

17. Grant PM, Kitch D, McComsey GA, Tierney C, Ha B, Brown TT. Differential skeletal impact of tenofovir disoproxil fumarate in young versus old HIV-infected adults. HIV Clin Trials. 2015;16(2):66-71.

18. Gupta SK, Mi D, Moe SM, Dube MP, Liu Z. Effects of switching from efavirenz to raltegravir on endothelial function, bone mineral metabolism, inflammation, and renal function: a randomized, controlled trial. J Acquir Immune Defic Syndr. 2013;64(3):279-83.

19. Moyle GJ, Stellbrink HJ, Compston J, et al. 96-Week results of abacavir/lamivudine versus tenofovir/ emtricitabine, plus efavirenz, in antiretroviralnaive, HIV-1-infected adults: ASSERT study. Antivir Ther. 2013;18(7):905-13.

20. Nelson MR, Elion RA, Cohen CJ, et al. Rilpivirine versus efavirenz in HIV-1-infected subjects receiving emtricitabine/tenofovir DF: pooled 96-week data from ECHO and THRIVE Studies. HIV Clin Trials. 2013;14(3):81-91.

21. Overton ET, Chan ES, Brown TT, et al. Vitamin D and calcium attenuate bone loss with antiretroviral therapy initiation: a randomized trial. Ann Intern Med. 2015;162(12):815-24.

22. Tebas P, Kumar P, Hicks C, et al. Greater change in bone turnover markers for efavirenz/emtricitabine/ tenofovir disoproxil fumarate versus dolutegravir + abacavir/lamivudine in antiretroviral therapy-naive adults over 144 weeks. AIDS. 2015;29(18):2459-64.

23. Wohl DA, Orkin C, Doroana M, et al. Change in vitamin $\mathrm{D}$ levels and risk of severe vitamin $\mathrm{D}$ deficiency over 48 weeks among HIV-1-infected, treatment-naive adults receiving rilpivirine or efavirenz in a Phase III trial (ECHO). Antivir Ther. 2014;19(2):191-200.

24. Sax PE, DeJesus E, Mills A, et al. Co-formulated elvitegravir, cobicistat, emtricitabine, and tenofovir versus co-formulated efavirenz, emtricitabine, and tenofovir for initial treatment of HIV-1 infection: a randomised, double-blind, phase 3 trial, analysis of results after 48 weeks. Lancet. 2012;379(9835):2439-48.

25. Zolopa A, Sax PE, DeJesus E, et al. A randomized double-blind comparison of coformulated elvitegravir/cobicistat/emtricitabine/tenofovir disoproxil fumarate versus efavirenz/emtricitabine/tenofovir disoproxil fumarate for initial treatment of HIV-1 infection: analysis of week 96 results. J Acquir Immune Defic Syndr. 2013;63(1):96-100. 
26. Bedimo R, Rosenblatt L, Myers J. Systematic review of renal and bone safety of the antiretroviral regimen efavirenz, emtricitabine, and tenofovir disoproxil fumarate in patients with HIV infection. HIV clinical trials. 2016;17(6):246-66.

27. Jullien V, Treluyer JM, Rey E, et al. Population pharmacokinetics of tenofovir in human immunodeficiency virus-infected patients taking highly active antiretroviral therapy. Antimicrob Agents Chemother. 2005;49(8):3361-6.

28. Gilead Sciences. Prescribing information for VIREAD $^{\circledR}$ (tenofovir disoproxil fumarate). http:// www.gilead.com/ /media/files/pdfs/medicines/ hiv/viread/viread_pi.pdf?la=en. Accessed 19 Oct 2017.

29. European Commission Health and Food Safety. Tybost Product Characteristics. Available at http:// ec.europa.eu/health/documents/communityregister/2015/20150210131020/anx_131020_en. pdf. Accessed 2 May 2016.

30. Hoetelmans R, Van Heeswijk R, Kestens Dea. Pharmacokinetic interaction between the novel nonnucleoside reverse transcriptase inhibitor (NNRTI) TMC278 and tenofovir disoproxil fumarate (TDF) in healthy volunteers. In: 3rd IAS conference on HIV pathogenesis, treatment and prevention, Brazil, July 2005, abstract WePe3.3C15.

31. Zimmermann AE, Pizzoferrato T, Bedford J, Morris A, Hoffman R, Braden G. Tenofovir-associated acute and chronic kidney disease: a case of multiple drug interactions. Clin Infect Dis. 2006;42(2):283-90.

32. Bristol Myers Squibb, Gilead Sciences. Prescribing information for ATRIPLA $^{\circledR}$ (efavirenz/tenofovir disoproxil fumarate/emtricitabine). http:// packageinserts.bms.com/pi/pi_atripla.pdf. Accessed 19 Oct 2017.

33. Gilead Sciences. Prescribing information for COMPLERA $^{\circledR}$ (rilpivirine/tenofovir disoproxil fumarate/ emtricitabine). https://gilead.com/ /media/files/ pdfs/medicines/hiv/complera/complera_pi.pdf. Accessed 19 Oct 2017.

34. Gilead Sciences. Prescribing information for STRIBILD $^{\circledR}$ (elvitegravir/cobicistat/tenofovir disoproxil fumarate/emtricitabine). http://www.gilead.com/ $\sim /$ media/files/pdfs/medicines/hiv/stribild/stribild_ pi.pdf?la=en. Accessed 19 Oct 2017.

35. Veterans Health Administration Office of Research and Development. Office of Research \& Development. Available from http://www.research.va.gov/ default.cfm. Accessed 24 Feb 2017.

36. Braithwaite RS, Kozal MJ, Chang CC, et al. Adherence, virological and immunological outcomes for
HIV-infected veterans starting combination antiretroviral therapies. AIDS. 2007;21(12):1579-89.

37. Gandhi NR, Tate JP, Rodriguez-Barradas MC, et al. Validation of an algorithm to identify antiretroviral-naive status at time of entry into a large, observational cohort of HIV-infected patients. Pharmacoepidemiol Drug Saf. 2013;22(9):1019-25.

38. LaFleur J, DuVall SL, Willson T, et al. Analysis of osteoporosis treatment patterns with bisphosphonates and outcomes among postmenopausal veterans. Bone. 2015;78:174-85.

39. LaFleur J, Ginter T, Curtis JR, et al. A novel method for obtaining bone mineral densities from a dataset of radiology reports and clinic notes: natural language processing in a national cohort of postmenopausal veterans. Presented at the 2013 annual meeting of the American Society for Bone and Mineral Research (ASBMR), Baltimore Convention Center, Baltimore, Maryland; 2013, October 4-7. http://www.asbmr.org/education/

AbstractDetail?aid=99cbf7b6-f3c3-49c4-9984-

Oba2962f1812. Accessed 13 Oct 2017.

40. Austin PC. Balance diagnostics for comparing the distribution of baseline covariates between treatment groups in propensity-score matched samples. Stat Med. 2009;28(25):3083-107.

41. Robins JM, Hernan MA, Brumback B. Marginal structural models and causal inference in epidemiology. Epidemiology. 2000;11(5):550-60.

42. Maggi P, Montinaro V, Mussini C, et al. Novel antiretroviral drugs and renal function monitoring of HIV patients. AIDS Rev. 2014;16(3):144-51.

43. Lepist EI, Zhang X, Hao J, et al. Contribution of the organic anion transporter OAT2 to the renal active tubular secretion of creatinine and mechanism for serum creatinine elevations caused by cobicistat. Kidney Int. 2014;86(2):350-7.

44. German P, Liu HC, Szwarcberg J, et al. Effect of cobicistat on glomerular filtration rate in subjects with normal and impaired renal function. J Acquir Immune Defic Syndr. 2012;61(1):32-40.

45. Tong L, Phan TK, Robinson KL, et al. Effects of human immunodeficiency virus protease inhibitors on the intestinal absorption of tenofovir disoproxil fumarate in vitro. Antimicrob Agents Chemother. 2007;51(10):3498-504.

46. Lepist EI, Phan TK, Roy A, et al. Cobicistat boosts the intestinal absorption of transport substrates, including HIV protease inhibitors and GS-7340, in vitro. Antimicrob Agents Chemother. 2012;56(10):5409-13. 
47. Ruane PJ, DeJesus E, Berger D, et al. Antiviral activity, safety, and pharmacokinetics/pharmacodynamics of tenofovir alafenamide as 10-day monotherapy in HIV-1-positive adults. J Acquir Immune Defic Syndr. 2013;63(4):449-55.

48. Pinheiro Edos S, Antunes OA, Fortunak JM. A survey of the syntheses of active pharmaceutical ingredients for antiretroviral drug combinations critical to access in emerging nations. Antiviral Res. 2008;79(3):143-65.

49. Ofotokun I, Titanji K, Lahiri CD, et al. A single-dose zoledronic acid infusion prevents antiretroviral therapy-induced bone loss in treatment-naive HIVinfected patients: a phase IIb trial. Clin Infect Dis. 2016;63(5):663-71.
50. Yin MT, Kendall MA, Wu X, et al. Fractures after antiretroviral initiation. AIDS. 2012;26(17):2175-84.

51. Triant VA, Brown TT, Lee H, Grinspoon SK. Fracture prevalence among human immunodeficiency virus (HIV)-infected versus non-HIV-infected patients in a large U.S. healthcare system. J Clin Endocrinol Metab. 2008;93(9):3499-504.

52. Bristol Myers Squibb. Prescribing information for SUSTIVA $^{\circledR}$ (efavirenz). http://packageinserts.bms. com/pi/pi_sustiva.pdf. Accessed 19 Oct 2017.

53. Brown MJ, Mezuk B. Brains, bones, and aging: psychotropic medications and bone health among older adults. Curr Osteoporos Rep. 2012;10(4):303-11. 